\title{
Soil Epidemiology in South West India
}

T Shaw

Melioidosis, caused by the soil saprophyte Burkholderiapseudomallei, is a potentially fatal tropical infection. The disease was under-diagnosed in India, as in many other South Asian countries; however, with increasing knowledge in recent times, there is an upscale in the number of diagnosed cases from different parts of the country. India might have the highest burden of the disease with an annual mortality of nearly 32,000 , as predicted recently, emphasizing that the disease should be considered an important public health issue in India.

Environmental surveillance detects the presence of the bacterium in specific geographical areas where humans are more prone to infection. It may help develop a pragmatic framework to develop a wide network for monitoring and evaluating case finding interventions, as well as to implement suitable policies for early detection, management and prevention of the disease.

Majority of the studies for environmental surveillance rely on culture based techniques which are laborious and have limited sensitivity. Hence a sensitive and prompt detection technique is a requisite to study the environmental influence on the persistence of the pathogen in the nature with various ecological factors in abundance. The South West coast of India is the home for tropical and subtropical moist broadleaf forest, high rainfall during the monsoon and rich organic soil, highly suitable for the persistence of the bacterium in the environment. Molecular approaches such as quantitative PCR techniques have shown promising results in understanding the distribution and burden of $B$. pseudomallei in the soil. The survey of B. pseudomalle $i$ in the soil of South West India is an approach to provide an ideal frame for environmental exploration of the bacterium in other parts of the country.

Department of Microbiology Kasturba Medical College Manipal. India .

Address for correspondence: Mr Tushar Shaw, Department of Microbiology, Kasturba Medical College, Manipal, India.+919008096827 Email: tusharshaw1990@gmail.com (ittps://orcid.org/0000-0002-6259-2107 